\title{
Assessment of Road Transport Atmospheric Emission of GHGs \& Criteria Pollutants in Qatar: BAU Versus Paris Agreement NDC Policy Scenarios
}

\author{
Azhari F. M. AHMED*; Lolwa AL- KUWARI; Aoubai KHAZNDAR and Abdulhadi AL MARRI \\ Climate Change Department, Ministry of Municipality \& Environment, Doha, Qatar
}

(30 August 2021, Doha, Qatar)

\begin{abstract}
The environmental impact of energy use on global climate change \& sustainable development, presents a serious challenge to modern societies. This is particularly true for road transport in fast developing countries like Qatar. Combustion of gasoline and diesel by road transport emits into the atmosphere direct greenhouse gases (GHGs) and air quality criteria pollutants (AQCPS). Scientists are now certain that GHGs, primarily carbon dioxide, methane and nitrous oxide are responsible for the observed global temperature increase which is projected to rise by approximately $1.7-3.9^{\circ} \mathrm{C}$ by 2100 . AQCPs which include carbon monoxide, Non-methane Volatile Organic Compounds, sulphur dioxide, particulate matter and oxides of nitrogen cause or contribute to local or regional air pollution with far reaching environmental impacts, notably on human health. There is now clear evidence of a causal relationship between exposure to traffic-related air pollution and health impacts such as exacerbation of asthma, non-asthma respiratory symptoms, impaired lung function and cardiovascular mortality and morbidity. Overall, the most problematic pollutants in terms of human health are particulate matter, especially PM2.5 and $\mathrm{O}_{3} . \mathrm{NO}_{2}$ is also a key concern because of both its direct health effects and its role as a precursor to ozone formation. Moreover, several AQCPs are recognized as short-lived climate forcing pollutants. Using fuel based emission factors developed by the International Panel for Climate Change \& the US Environmental Protection Agency, GHGs \& AQCPs emissions were estimated for the timeframe 1995-2020. IPCC based business as usual and policy emission-scenarios in line with Qatar's Nationally Determined Contribution under the Paris Agreement were investigated for the timeframe 2021-2030 to assess the potential emission reduction resulting from the mitigation measures which include e.g. launching of the now operational Doha electric railways network (Metro), battery-electrification of the public/private transport vehicles and introduction of EUR VI vehicle specifications. ${ }^{1}$
\end{abstract}

\section{Keywords}

Greenhouse gases, Air quality criteria pollutants, Nationally determined contribution, Human health, Climate Change

\section{Introduction}

The present investigation is partially based on a research project funded by Qatar Foundation (QF NPRP No.: 6-1035-5-126, 2015). The project's overall objective was to model the transition towards sustainability for a "smart" Doha City by providing a robust long-term planning model to help shape the future of Doha City energy use and utilization. It assesses the role of the interplay amongst smart technologies in a growing transport sector and within the context of climate change and air pollution. To complement the modeling exercise \& achieve the QF project goal, the lead author of this paper (co-investigator QF Project) made an assessment study of the emissions of GHGs \& Air Quality Criteria Pollutants (AQCPs) of Qatar's

* This paper is based on a contribution made by the lead author to a QF Research Project \# QF NPRP-6-1035-5126, 4 Oct. 2015; corresponding author e-mail address: afahmed@mme.gov.qa 
existing panorama of road transport fleet and also evaluated the impact of forecast scenarios up to 2035. The baseline information required to assess the environmental impact of the road transport sector in Qatar was obtained by the authors through contacts with the following entities: (i) Road Transport Division of the Ministry of Transport (https://www.motc.gov.qa/en/sectors/land-transport); (ii) Planning \& Statistics Authority, PSA(2021); and (iii) National Fuel Company-WOQOD (2019). Additional information was obtained from reviewing the sector's baseline data for 2007 published in Qatar's Initial National Communication to the UNFCCC (INC, Mo E, 2011).

The results discussed in this paper reflect an updated version of the original QF Project data sets to cover the period 2016-2020 \& projections up-to 2030. GHGs \& AQCPs-fuel based emission factors (EFs) for gasoline and diesel fueled vehicles were compiled from published reports by the International Panel for Climate Change (IPCC) \& US Environmental Protection Agency. Country specific tier-2 EFs were adapted using fuel-specific carbon contents \& heat values. Analysis of the baseline data covered vehicles fleet composition, traffic counts and the spatial \& temporal variations of traffic density in Doha. IPCC based business as usual and policy emission-scenarios in line with Qatar's Nationally Determined Contribution under the Paris Agreement were investigated to assess the potential emissions \& the reduction target. The potential policy measures to mitigate road transport air emissions in Qatar were outlined in Qatar's Initial Nationally Determined Contribution as part of the actions on adaptation-with-mitigation-benefits. They include existing \& planned measures e.g. launching of the now operational Doha electric railways network (Metro), battery-electrification of the public/private transport vehicles and introduction of EUR VI vehicle specifications. The paper discusses the chronology of road transport GHGs \& AQCPs emissions in Qatar and modeled projections based on BAU \& policy scenarios for the 2020-2030 timeframe. Emission intensities relative to socio-economic parameters were also partially examined.

\section{Data \& Methodology}

\subsection{Background}

Combustion of gasoline and diesel by road transport in Qatar emits into the atmosphere direct greenhouse gases (GHGs) and toxic air quality criteria pollutants (AQCPs). While the former group include carbon dioxide $\left(\mathrm{CO}_{2}\right)$, methane $\left(\mathrm{CH}_{4}\right)$ and nitrous oxide $\left(\mathrm{N}_{2} \mathrm{O}\right)$, the latter covers toxic air quality pollutants, known collectively as criteria pollutants i.e. carbon monoxide (CO), Non-methane Volatile Organic Compounds (NMVOCs), Sulphur dioxide $\left(\mathrm{SO}_{2}\right)$, particulate matter (PM) and oxides of nitrogen $\left(\mathrm{NO}_{\mathrm{x}}\right)$. These pollutants cause or contribute to local or regional air pollution with far reaching impacts on sustainable development, notably human health adverse effects (EU \& WHO, 2014). There is now clear evidence of a causal relationship between exposure to traffic-related air pollution and health impacts such as exacerbation of asthma, non-asthma respiratory symptoms, impaired lung function and cardiovascular mortality and morbidity. Overall, the strongest evidence for the most problematic pollutants in terms of human health is for particulate matter, especially PM2.5 and $\mathrm{O}_{3}$. $\mathrm{NO}_{2}$ is also a key concern because of both its direct health effects and the impact as a precursor to ozone formation (Hitchcock et. Al 2014). Road Transport includes all types of light duty vehicles such as automobile, light trucks and heavy-duty vehicles including tractor trailers and buses, and on-road motorcycles. Estimated emissions from road transport can be based on two independent approaches: fuel sale or vehicle distance covered in kilometer. If data sets are available for both approaches, it is important to check that they are comparable. While, the fuel-sale approach is appropriate for $\mathrm{CO}_{2}$, the second approach (based on distance travelled by vehicle type and road type) is suitable for $\mathrm{CH}_{4}, \mathrm{~N}_{2} \mathrm{O}$ and AQCPs. Generally, emissions from road transport fall into three groups: Exhaust emissions from the vehicle's engine as it is driven; Cold start emissions from the vehicle when started from cold and Evaporative fuel emissions from the vehicles' fuel system, engine and fuel tanks. For GHG inventory, the largest contribution comes from $\mathrm{CO}_{2}$. Evaporative emissions are not likely to be significant; they relate to NMVOC. A simple fuel-based approach will estimate emissions of $\mathrm{CO}_{2}$ accurately enough 
provided that the fuel consumption statistics are known. A similar approach based on published emission factors can be used for other gases where detailed data is not available.

Generally, estimation of emissions from road transport is possible at three tier levels including a default tier 1 approach; country specific tier 2 and source specific tier 3 . The data requirements for tier 3 methodology, which represents the most accurate approach, includes a wide range of parameters i.e. fuel consumption, quality of fuels used, emission controls fitted to vehicle, operating characteristics (e.g. average vehicle speeds or types of road), maintenance record, fleet age distribution, distance driven and climate (IPCC 2000, Good Practice Guidance). Usually, not all of these data are available; for e.g. total fuel consumption may be known but not disaggregated by type of vehicles (e.g. total petrol sales, but not petrol consumption by cars, light-duty trucks and motorcycles separately). The simplest methodology, tier 1 , is based on fuel consumption. If the fuel's carbon content is known, then tier 2 may be employed to determine $\mathrm{CO}_{2}$ emissions more accurately. The $\mathrm{CO}_{2}$ emission factor considers all the carbon in the fuel including that emitted as $\mathrm{CO}_{2}, \mathrm{CH}_{4}, \mathrm{CO}, \mathrm{NMVOC}$ and particulate matter.

In general, the best estimates (tier 3 ) for emissions from vehicles may be summarized by the following equation:

Emissions $=\sum_{a b c d}\left(E_{a b c d} *\right.$ Activity $\left._{a b c d}\right)+\sum_{b}$ Cold $_{b}+\sum_{b}$ Evaporation $_{b}$

Where:

Emission: Total emissions from road transport; EF: Emission factor, as mass per unit of activity rate; Activity: activity rate (fuel consumed, or distance travelled); Cold: Extra emissions due to cold starts; Evaporation: extra emissions due to evaporation (NMVOC) ; a: fuel type (petrol, diesel, LPG, etc.) ; b: vehicle type (passenger car, light-duty truck, bus, etc.); c: emission control; d: road type or vehicle speed.

\subsection{Available Baseline Information}

At the present, the road transport data available in Qatar is limited and not detailed enough to allow computation of the emissions at the tier 3 level described in the equation above. Since acquiring and collating such data requires commissioning of extensive surveys and analysis beyond the means of the authors, the baseline data used in this report is limited and allows assessment at the tier 1-2 level at its best. The baseline data and sources of information considered in this paper were obtained from: Qatar Planning \& Statistics Authority, PSA (2021); \& the national fuel company WOQOD (2019)

. Gasoline and diesel fuel sales statistics covering the period 1995-2020 were obtained from WOQOD in addition to fuel specifications. Since the available diesel sales statistics for the updated period (2016 to 2020) were not disaggregated between the vehicular and project use in non-road equipment e.g. stationary gas turbines \& other heavy duty construction equipment a universal ratio of $\sim 40 \%$ was used to estimate the road transport diesel from the total diesel statistics published by WOQOD. This ratio was based on the earlier data split and a personal communication with the company.

\subsection{Emission Factors}

Fuel based emission factors (EFs) for gasoline and diesel fueled passenger cars, light duty \& heavy duty vehicles manufactured in USA \& Europe were compiled from published reports by the International Panel for Climate Change \& US Environmental Protection Agency (Revised IPCC Guidelines 1996; 2006 IPCC Guidelines; IPCC Good Practice Guidance 2000; USEPA 1985). Table 1 shows a compilation of the EFs and the aggregated coefficients used for estimating emissions. For all vehicle categories and fuels, uncontrolled emission factors were selected. Hence the AQCPs and $\mathrm{CH} 4$ estimates are probably slightly higher in case emission control technology is employed by some of the new models. $\mathrm{CO}_{2} \& \mathrm{~N}_{2} \mathrm{O}$ emissions, on the other hand, are independent of catalyst and non- catalyst controls and their estimates are relatively more accurate. Since $\mathrm{CO}_{2}$ is estimated on basis of country specific fuel calorific values the expected uncertainty is low probably < 10\% (EU ETS Guidelines 2007). Another limitation in the EFs is that they cover only vehicles manufactured in USA \& Europe and do not include Japanese vehicles, which constitute a large fraction of the Qatari fleet. It has been assumed that the latter brand is intermediate in its emissions 
relative to the other two classes of vehicles and hence will not impact the estimated emissions (ECMT 2000).

\begin{tabular}{|c|c|c|c|c|c|c|c|c|}
\hline $\begin{array}{c}\text { Emission } \\
\text { Source }\end{array}$ & $\mathrm{CO}_{2}$ & $\mathrm{CH}_{4}$ & $\mathrm{~N}_{2} \mathrm{O}$ & $\mathrm{NMHC}$ & $\mathrm{CO}$ & $\mathrm{PM} 10$ & $\mathrm{NO}_{x}$ & $\mathrm{SO}_{2}$ \\
\hline $\begin{array}{c}\text { Gasoline } \\
\text { US pass cars }\end{array}$ & 72.1 & $0.02(0.03)^{* *}$ & 0.003 & 0.93 & 4.83 & 0.40 & 0.22 & 0.006 \\
\hline $\begin{array}{c}\text { Gasoline } \\
\text { US LDT }\end{array}$ & 72.1 & $0.02(0.03)^{* *}$ & 0.003 & 0.96 & 4.48 & 0.35 & 0.23 & 0.006 \\
\hline $\begin{array}{c}\text { Gasoline } \\
\text { EU Pass cars }\end{array}$ & 73.0 & 0.02 & 0.001 & 1.5 & 13.0 & 0.40 & 0.60 & 0.006 \\
\hline $\begin{array}{c}\text { Gasoline } \\
\text { EU LDT }\end{array}$ & 73.0 & 0.02 & 0.001 & 1.4 & 8.3 & 0.35 & 0.70 & 0.006 \\
\hline $\begin{array}{c}\text { Gasoline } \\
\text { Aggregate EFs }\end{array}$ & 72.5 & 0.02 & 0.002 & 1.2 & 7.65 & 0.38 & 0.44 & 0.006 \\
\hline Diesel \\
US HDT
\end{tabular}

Table 1. Compilation of Gasoline and Diesel Mobile Source Emission Factors in t/TJ

* US LDT = US light duty trucks; US HDT = US heavy duty trucks; EULDT, EU HDT = European light duty and heavy-duty trucks.

** EFs in brackets IPCC 2006

\subsection{Results \& Discussion}

\subsection{Fleet Composition \& Traffic Counts}

Composition of the road transport fleet in Qatar is one of the most important parameters which determine the overall level of emissions from the sector. Traffic counts particularly in terms of the spatial and temporal variations of the traffic density provide an insight on the emission strength of air pollutants \& the potential impact on air quality and human health. Analysis of the historical accumulative registration statistics of vehicles and motorcycles in Qatar suggests that private passenger vehicles constitute the bulk of the road transport sector, ranging from $65 \%$ to $67 \%$ of the total fleet and growing annually between $5 \%$ $15 \%$ during 1998-2013. It tallied approximately over 600,000 vehicles in 2013. Private business transport, which consists of buses and light duty trucks represents the second largest mode of transport, ranging between 25 and $30 \%$ of the total fleet (approximately a quarter of million vehicles in 2013). Heavy mobile equipment represents $2-3 \%$ of the fleet or approximately 25000 vehicles. Taxis which enjoyed the highest growth rate of the road transport sector between 1997 and 2013 (1500\%) represent 3\% of the mobile fleet. Motorcycles, represents $1-2 \%$ and public transport 1\%. Government \& Others represent $<1 \%$. Overall, during 2004-2009 road transport witnessed the highest growth rate ranging between $10 \%$ \& $16 \%$. 


\subsection{Chronology of Fuel Consumption \& Annual Emissions}

Estimates of road transport annual air emissions computed from fuel consumption statistics (Table 2) and aggregated emission factors (Table 1 ) are shown in Table 3. Emission of $\mathrm{GHGs}\left(\mathrm{CO}_{2}, \mathrm{CH}_{4}, \mathrm{~N}_{2} \mathrm{O}\right)$ during the period 1995-2015 has also been computed in $\mathrm{CO}_{2}$ equivalence using IPCC global warming potentials of $\mathrm{CO}_{2}$ (1); $\mathrm{CH}_{4}$ (21) and $\mathrm{N}_{2} \mathrm{O}$ (310) (IPCC 2007). Estimates for $\mathrm{AQCPs}$ emissions of $\mathrm{NO}_{x}, \mathrm{NMHC}, \mathrm{CO}$ and $\mathrm{SO}_{2}$ were calculated separately and as annual totals. The emission results were also disaggregated to indicate both the split between the diesel and gasoline powered vehicles and the evolution of emission with time. The results suggest that between 1995 and 2015/2020, the total GHG emissions from road transport has grown by approximately five times from under 2 million to about 9 million ton. Likewise, the total AQCP emissions increased from about 200,000 to 900,000 ton. Overall, gasoline powered vehicles were responsible for approximately $70 \%$ \& $90 \%$, respectively, of the total emissions of GHGs and AQCPs, the rest $30 \% \& 10 \%$ are due to combustion of diesel fuel. While $\mathrm{CO}_{2}$ constitutes approximately $>98 \%$ of the GHG emissions; $\mathrm{NO}_{x}, \mathrm{NMHC}, \mathrm{CO}, \mathrm{PM} 10$ and $\mathrm{SO}_{2}$ represent $8 \%, 11 \%, 74 \%, 6 \%$ and $<1 \%$ of the total AQCP, respectively. The emission trend of GHGs \& AQCPs from road transport in Qatar follows generally the growth in the GDP. During 1995-2004 emissions increased modestly, approximately at 4-6\% annually. This was followed by a drastic increase reaching approximately 20\% during 2004-2007 and peaking at $>35 \%$ in 2008. During 2009-2012 the growth in emissions receded to a lower level, about 2-4\% followed by an increase from 2013 to 2015 ranging 8\% to 14\%. During the updated period from 2016 to 2020, the growth in emissions continued at a modest rate ranging between 3-8\% during 2016 \& 2017 and then decreased gradually by about $-10 \%$ for both GHGs \& AQCPs between 2019-2020. The drop in emissions was due to the COVID-19 Pandemic. The historic evolution of car registration \& air emissions in Qatar is shown in Table 3 \& Figure 1.

\begin{tabular}{|c|c|c|c|c|c|c|c|}
\hline Year & $\begin{array}{c}\text { Total } \\
\text { Gasoline }\end{array}$ & Diesel & $\begin{array}{c}\text { Total Energy } \\
\text { Use }\end{array}$ & $\begin{array}{c}\text { Road Transport } \\
\text { Diesel }\end{array}$ & $\begin{array}{l}\mathrm{CO}_{2} \mathrm{Eq} . \\
\text { Emission }\end{array}$ & $\begin{array}{c}\text { GDP } \\
\text { Current } \\
\text { USD }\end{array}$ & Emission/GDP \\
\hline & \multicolumn{3}{|c|}{ TJ } & $\%$ & $\mathrm{Kt}$ & Billion \$ & $\begin{array}{c}\mathrm{t}-\mathrm{CO}_{2} \text { eq. Per } \\
\text { Million US\$ }\end{array}$ \\
\hline 1995 & 18,234 & 11,804 & 30,038 & 39 & 1,856 & 16 & 116 \\
\hline 1996 & 18,548 & 12,873 & 31,422 & 41 & 1,936 & 17 & 114 \\
\hline 1997 & 19,700 & 15,356 & 35,056 & 44 & 2,131 & 21 & 101 \\
\hline 1998 & 20,545 & 15,777 & 36,321 & 43 & 2,212 & 19 & 112 \\
\hline 1999 & 21,086 & 12,491 & 33,577 & 37 & 2,038 & 22 & 92 \\
\hline 2000 & 22,093 & 13,370 & 35,463 & 38 & 2,148 & 17.8 & 121 \\
\hline 2001 & NA & NA & NA & 40 & 2,245 & 19 & 118 \\
\hline 2002 & NA & NA & NA & 40 & 2,341 & 19.4 & 121 \\
\hline 2003 & NA & 21,850 & NA & 40 & 3,084 & 26 & 119 \\
\hline 2004 & 26,967 & 26,664 & 53,630 & 40 & 2,688 & 31.7 & 85 \\
\hline 2005 & NA & NA & NA & 40 & 3,925 & 46 & 85 \\
\hline 2006 & NA & NA & NA & 40 & 5,163 & 60.9 & 85 \\
\hline
\end{tabular}


Ahmed, A. F. M.; Al-Kuwari, L;

Khazndar, A; Al-Marri, A

Assessment of Road Transport Atmospheric Emission of GHGs \& Criteria Pollutants

in Qatar: BAU Versus Paris

Agreement NDC Policy Scenarios

\begin{tabular}{||c||c|c|c||c||c|c||c||}
\hline 2007 & 36,605 & 25,126 & 61,731 & 41 & 4,435 & 79.4 & 56 \\
\hline 2008 & 47,796 & 33,647 & 81,443 & 41 & 6,008 & 115.8 & 52 \\
\hline 2009 & 50,215 & 32,852 & 83,067 & 40 & 6,127 & 97.8 & 63 \\
\hline 2010 & 53,500 & 32,317 & 85,817 & 38 & 6,329 & 125.1 & 51 \\
\hline 2011 & 57,327 & 31,584 & 88,910 & 36 & 6,556 & 167.8 & 39 \\
\hline 2012 & 56,677 & 31,568 & 88,245 & 36 & 6,507 & 186.8 & 35 \\
\hline 2013 & 69,420 & 31,599 & 101,019 & 31 & 7,445 & 198.7 & 37 \\
\hline 2014 & 76,604 & 35,602 & 112,207 & 32 & 8,271 & 206.2 & 40 \\
\hline $2015 *$ & 81,694 & 39,504 & 121,198 & 33 & 8,934 & 161.7 & 55 \\
\hline 2016 & 86,640 & 44312 & 130952 & 40 & 9,653 & 151.7 & 64 \\
\hline 2017 & 90,250 & 44312 & 134562 & 40 & 9,908 & 161.1 & 62 \\
\hline 2018 & 92,199 & 43120 & 135319 & 40 & 9,942 & 183.3 & 54 \\
\hline 2019 & 95,196 & 39545 & 134741 & 40 & 9,858 & 168 & 59 \\
\hline 2020 & 86,640 & 35144 & 121784 & 40 & 8,913 & 146.4 & 60 \\
\hline
\end{tabular}

Table 2. Baseline Road Transport Historical Fuel Consumption Statistic \& BAU GHG Emissions 1995-2020

\begin{tabular}{|c|c|c|c|c|c|}
\hline Years & Items & $\begin{array}{l}\text { Gasoline } \\
(\mathrm{T})\end{array}$ & $\begin{array}{c}\text { Diesel } \\
(\text { Road + HDE) }\end{array}$ & Grand Total & $\begin{array}{c}\text { Change } \\
\text { /Year } \\
(\%) \\
\end{array}$ \\
\hline \multirow{10}{*}{1995} & $\mathrm{CO} 2(\mathrm{kt})$ & 1313.0 & 517.0 & 1830.0 & - \\
\hline & $\mathrm{CH} 4(\mathrm{t})$ & 365.0 & 35.0 & 400.0 & - \\
\hline & $\mathrm{N} 2 \mathrm{O}(\mathrm{t})$ & 36.0 & 21.0 & 57.0 & - \\
\hline & $\mathrm{CO}_{2} \mathrm{Eq} .(\mathrm{t})$ & 1331825 & 524245 & 1856070 & - \\
\hline & $\mathrm{NOx}(\mathrm{t})$ & 8020.0 & 6020.0 & 14040.0 & - \\
\hline & $\mathrm{NMHC}(\mathrm{t})$ & 21880.0 & 1060.0 & 22940.0 & - \\
\hline & $\mathrm{CO}(\mathrm{t})$ & 139490.0 & 4320.0 & 143810.0 & - \\
\hline & PM-10 (t) & 6930.0 & 4390.0 & 11320.0 & - \\
\hline & $\mathrm{SO} 2(\mathrm{t})$ & 110.0 & 155.0 & 265.0 & - \\
\hline & Total AQCP (t) & 176430 & 15945 & 192375 & - \\
\hline \multirow{8}{*}{1996} & $\mathrm{CO} 2(\mathrm{kt})$ & 1345.0 & 564.0 & 1909.0 & $4 \%$ \\
\hline & $\mathrm{CH} 4(\mathrm{t})$ & 371.0 & 38.0 & 409.0 & $2 \%$ \\
\hline & $\mathrm{N} 2 \mathrm{O}(\mathrm{t})$ & 37.0 & 23.0 & 60.0 & $5 \%$ \\
\hline & $\mathrm{CO}_{2}$ Eq. $(\mathrm{t})$ & 1364261 & 571928 & 1936189 & $4 \%$ \\
\hline & $\mathrm{NOx}(\mathrm{t})$ & 8160.0 & 6565.0 & 14725.0 & $5 \%$ \\
\hline & $\mathrm{NMHC}(\mathrm{t})$ & 22258.0 & 1159.0 & 23417.0 & $2 \%$ \\
\hline & $\mathrm{CO}(\mathrm{t})$ & 141892.0 & 4709.0 & 146601.0 & $2 \%$ \\
\hline & PM-10 (t) & 7048.0 & 4785.0 & 11833.0 & $5 \%$ \\
\hline
\end{tabular}


Ahmed, A. F. M.; Al-Kuwari, L;

Khazndar, A; Al-Marri, A

Assessment of Road Transport Atmospheric

Emission of GHGs \& Criteria Pollutants

in Qatar: BAU Versus Paris

Agreement NDC Policy Scenarios

\begin{tabular}{|c|c|c|c|c|c|}
\hline & $\mathrm{SO} 2(\mathrm{t})$ & 112.0 & 170.0 & 282.0 & $6 \%$ \\
\hline & Total AQCP (t) & 179470 & 17388 & 196858 & $2 \%$ \\
\hline \multirow{10}{*}{1997} & $\mathrm{CO} 2(\mathrm{kt})$ & 1428.0 & 673.0 & 2101.0 & $10 \%$ \\
\hline & $\mathrm{CH} 4(\mathrm{t})$ & 394.0 & 46.0 & 440.0 & $8 \%$ \\
\hline & $\mathrm{N} 2 \mathrm{O}(\mathrm{t})$ & 39.0 & 28.0 & 67.0 & $12 \%$ \\
\hline & $\mathrm{CO}_{2} \mathrm{Eq} \cdot(\mathrm{t})$ & 1448364 & 682646 & 2131010 & $10 \%$ \\
\hline & $\mathrm{NOx}(\mathrm{t})$ & 8668.0 & 7832.0 & 16500.0 & $12 \%$ \\
\hline & $\mathrm{NMHC}(\mathrm{t})$ & 23640.0 & 1382.0 & 25022.0 & $7 \%$ \\
\hline & $\mathrm{CO}(\mathrm{t})$ & 150705.0 & 5621.0 & 156326.0 & $7 \%$ \\
\hline & PM-10 (t) & 7486.0 & 5713.0 & 13199.0 & $12 \%$ \\
\hline & $\mathrm{SO} 2(\mathrm{t})$ & 118.0 & 203.0 & 321.0 & $14 \%$ \\
\hline & Total AQCP (t) & 190617 & 20751 & 211368 & $7 \%$ \\
\hline \multirow{10}{*}{1998} & $\mathrm{CO} 2(\mathrm{kt})$ & 1489.5 & 691.5 & 2181.0 & $4 \%$ \\
\hline & $\mathrm{CH} 4(\mathrm{t})$ & 411.0 & 47.0 & 458.0 & $4 \%$ \\
\hline & $\mathrm{N} 2 \mathrm{O}(\mathrm{t})$ & 41.0 & 28.0 & 69.0 & $3 \%$ \\
\hline & $\mathrm{CO}_{2}$ Eq. (t) & 1510841 & 701167 & 2212008 & $4 \%$ \\
\hline & $\mathrm{NOx}(\mathrm{t})$ & 9040.0 & 8046.0 & 17086.0 & $4 \%$ \\
\hline & $\mathrm{NMHC}(\mathrm{t})$ & 24653.0 & 2366.0 & 27019.0 & $8 \%$ \\
\hline & $\mathrm{CO}(\mathrm{t})$ & 157165.0 & 5774.0 & 162939.0 & $4 \%$ \\
\hline & PM-10 (t) & 7807.0 & 5869.0 & 13676.0 & $4 \%$ \\
\hline & $\mathrm{SO} 2(\mathrm{t})$ & 123.0 & 208.0 & 331.0 & $3 \%$ \\
\hline & Total AQCP (t) & 198788.0 & 22263.0 & 221051.0 & $5 \%$ \\
\hline \multirow{10}{*}{1999} & $\mathrm{CO} 2(\mathrm{kt})$ & 1461.2 & 547.5 & 2008.7 & $-8 \%$ \\
\hline & $\mathrm{CH} 4(\mathrm{t})$ & 422.0 & 37.0 & 459.0 & $0 \%$ \\
\hline & $\mathrm{N} 2 \mathrm{O}(\mathrm{t})$ & 42.0 & 22.5 & 64.5 & $-7 \%$ \\
\hline & $\mathrm{CO}_{2} \mathrm{Eq} \cdot(\mathrm{t})$ & 1483082.0 & 555252.0 & 2038334.0 & $-8 \%$ \\
\hline & $\mathrm{NOx}(\mathrm{t})$ & 9278.0 & 6371.0 & 15649.0 & $-8 \%$ \\
\hline & $\mathrm{NMHC}(\mathrm{t})$ & 25303.0 & 1124.0 & 26427.0 & $-2 \%$ \\
\hline & $\mathrm{CO}(\mathrm{t})$ & 161308.0 & 4572.0 & 165880.0 & $2 \%$ \\
\hline & PM-10 (t) & 8013.0 & 4647.0 & 12660.0 & $-7 \%$ \\
\hline & $\mathrm{SO} 2(\mathrm{t})$ & 127.0 & 165.0 & 292.0 & $-12 \%$ \\
\hline & Total AQCP (t) & 204029.0 & 16879.0 & 220908.0 & $0 \%$ \\
\hline \multirow{10}{*}{2000} & $\mathrm{CO} 2(\mathrm{kt})$ & 1531.0 & 586.0 & 2117.0 & $5 \%$ \\
\hline & $\mathrm{CH} 4(\mathrm{t})$ & 442.0 & 40.0 & 482.0 & $5 \%$ \\
\hline & $\mathrm{N} 2 \mathrm{O}(\mathrm{t})$ & 44.0 & 24.0 & 68.0 & $5 \%$ \\
\hline & $\mathrm{CO}_{2} \mathrm{Eq} .(\mathrm{t})$ & 1553922.0 & 594280.0 & 2148202.0 & $5 \%$ \\
\hline & $\mathrm{NOx}(\mathrm{t})$ & 9721.0 & 6819.0 & 16540.0 & $6 \%$ \\
\hline & $\mathrm{NMHC}(\mathrm{t})$ & 26512.0 & 1203.0 & 27715.0 & $5 \%$ \\
\hline & $\mathrm{CO}(\mathrm{t})$ & 169011.0 & 4893.0 & 173904.0 & $5 \%$ \\
\hline & PM-10 (t) & 8395.0 & 4974.0 & 13369.0 & $6 \%$ \\
\hline & $\mathrm{SO} 2(\mathrm{t})$ & 133.0 & 176.5 & 309.5 & $6 \%$ \\
\hline & Total AQCP (t) & 213772.0 & 18065.5 & 231837.5 & $5 \%$ \\
\hline 2004 & $\mathrm{CO} 2(\mathrm{kt})$ & 1869.5 & 779.0 & 2648.5 & $25 \%$ \\
\hline
\end{tabular}


Ahmed, A. F. M.; Al-Kuwari, L;

Khazndar, A; Al-Marri, A

Assessment of Road Transport Atmospheric

Emission of GHGs \& Criteria Pollutants

in Qatar: BAU Versus Paris

Agreement NDC Policy Scenarios

\begin{tabular}{|c|c|c|c|c|c|}
\hline & $\mathrm{CH} 4(\mathrm{t})$ & 540.0 & 53.0 & 593.0 & $23 \%$ \\
\hline & $\mathrm{N} 2 \mathrm{O}(\mathrm{t})$ & 54.0 & 32.0 & 86.0 & $26 \%$ \\
\hline & $\mathrm{CO}_{2} \mathrm{Eq} \cdot(\mathrm{t})$ & 1897580.0 & 790033.0 & 2687613.0 & $25 \%$ \\
\hline & $\mathrm{NOx}(\mathrm{t})$ & 11870.0 & 9066.0 & 20936.0 & $27 \%$ \\
\hline & $\mathrm{NMHC}(\mathrm{t})$ & 32374.0 & 1600.0 & 33974.0 & $23 \%$ \\
\hline & $\mathrm{CO}(\mathrm{t})$ & 206382.0 & 6506.0 & 212888.0 & $22 \%$ \\
\hline & PM-10 (t) & 10252.0 & 6613.0 & 16865.0 & $26 \%$ \\
\hline & $\mathrm{SO} 2(\mathrm{t})$ & 162.0 & 234.7 & 396.7 & $28 \%$ \\
\hline & Total AQCP (t) & 261040.0 & 24019.7 & 285059.7 & $23 \%$ \\
\hline \multirow{10}{*}{2007} & $\mathrm{CO} 2(\mathrm{kt})$ & 2536.5 & 1835.0 & 4371.5 & $65 \%$ \\
\hline & $\mathrm{CH} 4(\mathrm{t})$ & 732.0 & 125.0 & 857.0 & $45 \%$ \\
\hline & $\mathrm{N} 2 \mathrm{O}(\mathrm{t})$ & 73.0 & 75.0 & 148.0 & $72 \%$ \\
\hline & $\mathrm{CO}_{2}$ Eq. (t) & 2574502.0 & 1860875.0 & 4435377.0 & $65 \%$ \\
\hline & $\mathrm{NOx}(\mathrm{t})$ & 16104.0 & 21357.0 & 37461.0 & $79 \%$ \\
\hline & $\mathrm{NMHC}(\mathrm{t})$ & 43922.0 & 2764.0 & 46686.0 & $37 \%$ \\
\hline & $\mathrm{CO}(\mathrm{t})$ & 280000.0 & 15327.0 & 295327.0 & $39 \%$ \\
\hline & PM-10 (t) & 13909.0 & 15578.0 & 29487.0 & $75 \%$ \\
\hline & $\mathrm{SO} 2(\mathrm{t})$ & 220.0 & 553.0 & 773.0 & $95 \%$ \\
\hline & Total AQCP (t) & 354155.0 & 55579.0 & 409734.0 & $44 \%$ \\
\hline \multirow{10}{*}{2008} & $\mathrm{CO} 2(\mathrm{kt})$ & 3465.2 & 2457.9 & 5923.1 & $35 \%$ \\
\hline & $\mathrm{CH} 4(\mathrm{t})$ & 955.9 & 168.2 & 1124.2 & $31 \%$ \\
\hline & $\mathrm{N} 2 \mathrm{O}(\mathrm{t})$ & 95.6 & 100.9 & 196.5 & $33 \%$ \\
\hline & $\mathrm{CO}_{2} \mathrm{Eq} .(\mathrm{t})$ & 3514947.3 & 2492701.0 & 6007648.2 & $35 \%$ \\
\hline & $\mathrm{NOx}(\mathrm{t})$ & 21030.4 & 28599.5 & 49629.9 & $32 \%$ \\
\hline & $\mathrm{NMHC}(\mathrm{t})$ & 57355.7 & 5047.0 & 62402.7 & $34 \%$ \\
\hline & $\mathrm{CO}(\mathrm{t})$ & 365642.5 & 20524.4 & 386166.8 & $31 \%$ \\
\hline & PM-10 (t) & 18162.6 & 20860.8 & 39023.5 & $32 \%$ \\
\hline & $\mathrm{SO} 2(\mathrm{t})$ & 286.8 & 740.2 & 1027.0 & $33 \%$ \\
\hline & Total AQCP (t) & 462478.0 & 75771.9 & 538249.9 & $31 \%$ \\
\hline \multirow{10}{*}{2009} & $\mathrm{CO} 2(\mathrm{kt})$ & 3640.6 & 2399.8 & 6040.4 & $2 \%$ \\
\hline & $\mathrm{CH} 4(\mathrm{t})$ & 1004.3 & 164.3 & 1168.6 & $4 \%$ \\
\hline & $\mathrm{N} 2 \mathrm{O}(\mathrm{t})$ & 100.4 & 98.6 & 199.0 & $1 \%$ \\
\hline & $\mathrm{CO}_{2} \mathrm{Eq} \cdot(\mathrm{t})$ & 3692811.1 & 2433840.4 & 6126651.5 & $2 \%$ \\
\hline & $\mathrm{NOx}(\mathrm{t})$ & 22094.6 & 27924.2 & 50018.8 & $1 \%$ \\
\hline & $\mathrm{NMHC}(\mathrm{t})$ & 64200.0 & 4927.8 & 69127.8 & $11 \%$ \\
\hline & $\mathrm{CO}(\mathrm{t})$ & 438551.6 & 20039.7 & 458591.3 & $19 \%$ \\
\hline & PM-10 (t) & 21537.3 & 20368.2 & 41905.5 & $7 \%$ \\
\hline & $\mathrm{SO} 2(\mathrm{t})$ & - & 722.7 & 722.7 & $-30 \%$ \\
\hline & Total AQCP (t) & 546383.4 & 73982.7 & 620366.1 & $15 \%$ \\
\hline \multirow{4}{*}{2010} & $\mathrm{CO} 2(\mathrm{kt})$ & 3878.8 & 2360.8 & 6239.5 & $3 \%$ \\
\hline & $\mathrm{CH} 4(\mathrm{t})$ & 1070.0 & 161.6 & 1231.6 & $5 \%$ \\
\hline & $\mathrm{N} 2 \mathrm{O}(\mathrm{t})$ & 107.0 & 97.0 & 204.0 & $2 \%$ \\
\hline & $\mathrm{CO}_{2} \mathrm{Eq} \cdot(\mathrm{t})$ & 3934390.0 & 2394219.8 & 6328609.8 & $3 \%$ \\
\hline
\end{tabular}


Ahmed, A. F. M.; Al-Kuwari, L;

Khazndar, A; Al-Marri, A

Assessment of Road Transport Atmospheric

Emission of GHGs \& Criteria Pollutants

in Qatar: BAU Versus Paris

Agreement NDC Policy Scenarios

\begin{tabular}{|c|c|c|c|c|c|}
\hline & $\mathrm{NOx}(\mathrm{t})$ & 23540.0 & 27469.6 & 51009.6 & $2 \%$ \\
\hline & $\mathrm{NMHC}(\mathrm{t})$ & 64200.0 & 4847.6 & 69047.6 & $0 \%$ \\
\hline & $\mathrm{CO}(\mathrm{t})$ & 409275.0 & 19713.5 & 428988.5 & $-6 \%$ \\
\hline & PM-10 (t) & 20330.0 & 20036.7 & 40366.7 & $-4 \%$ \\
\hline & $\mathrm{SO} 2(\mathrm{t})$ & 321.0 & 711.0 & 1032.0 & $43 \%$ \\
\hline & Total AQCP (t) & 517666.0 & 72778.3 & 590444.3 & $-5 \%$ \\
\hline \multirow{10}{*}{2011} & $\mathrm{CO} 2(\mathrm{kt})$ & 4156.2 & 2307.2 & 6463.4 & $4 \%$ \\
\hline & $\mathrm{CH} 4(\mathrm{t})$ & 1146.5 & 157.9 & 1304.5 & $6 \%$ \\
\hline & $\mathrm{N} 2 \mathrm{O}(\mathrm{t})$ & 114.7 & 94.8 & 209.4 & $3 \%$ \\
\hline & $\mathrm{CO}_{2}$ Eq. $(\mathrm{t})$ & 4215827.6 & 2339871.0 & 6555698.6 & $4 \%$ \\
\hline & $\mathrm{NOx}(\mathrm{t})$ & 25223.9 & 26846.1 & 52069.9 & $2 \%$ \\
\hline & $\mathrm{NMHC}(\mathrm{t})$ & 68792.4 & 4737.5 & 73529.9 & $6 \%$ \\
\hline & $\mathrm{CO}(\mathrm{t})$ & 438551.6 & 19266.0 & 457817.5 & $7 \%$ \\
\hline & PM-10 (t) & 21784.3 & 19581.8 & 41366.1 & $2 \%$ \\
\hline & $\mathrm{SO} 2(\mathrm{t})$ & 344.0 & 694.8 & 1038.8 & $1 \%$ \\
\hline & Total AQCP $(t)$ & 554696.1 & 71126.3 & 625822.3 & $6 \%$ \\
\hline \multirow{10}{*}{2012} & $\mathrm{CO} 2(\mathrm{kt})$ & 4109.1 & 2306.1 & 6415.2 & $-1 \%$ \\
\hline & $\mathrm{CH} 4(\mathrm{t})$ & 1133.5 & 157.8 & 1291.4 & $-1 \%$ \\
\hline & $\mathrm{N} 2 \mathrm{O}(\mathrm{t})$ & 113.4 & 94.7 & 208.1 & $-1 \%$ \\
\hline & CO2 Eq. (t) & 4168026.6 & 2338744.9 & 6506771.5 & $-1 \%$ \\
\hline & $\mathrm{NOx}(\mathrm{t})$ & 24937.9 & 26833.1 & 51771.0 & $-1 \%$ \\
\hline & $\mathrm{NMHC}(\mathrm{t})$ & 68012.4 & 4735.3 & 72747.7 & $-1 \%$ \\
\hline & $\mathrm{CO}(\mathrm{t})$ & 433579.1 & 19256.7 & 452835.8 & $-1 \%$ \\
\hline & PM-10 (t) & 21537.3 & 19572.4 & 41109.7 & $-1 \%$ \\
\hline & $\mathrm{SO} 2(\mathrm{t})$ & 340.1 & 694.5 & 1034.6 & $0 \%$ \\
\hline & Total AQCP (t) & 548406.7 & 71092.0 & 619498.7 & $-1 \%$ \\
\hline \multirow{10}{*}{2013} & $\mathrm{CO} 2(\mathrm{kt})$ & 5031.6 & 2308.3 & 7339.9 & $14 \%$ \\
\hline & $\mathrm{CH} 4(\mathrm{t})$ & 1388.0 & 158.0 & 1546.0 & $20 \%$ \\
\hline & $\mathrm{N} 2 \mathrm{O}(\mathrm{t})$ & 138.8 & 94.8 & 233.6 & $12 \%$ \\
\hline & CO2 Eq. (t) & 5103809.6 & 2341014.9 & 7444824.5 & $14 \%$ \\
\hline & $\mathrm{NOx}(\mathrm{t})$ & 30536.8 & 26859.2 & 57396.0 & $11 \%$ \\
\hline & $\mathrm{NMHC}(\mathrm{t})$ & 83282.2 & 4739.9 & 88022.0 & $21 \%$ \\
\hline & $\mathrm{CO}(\mathrm{t})$ & 530923.9 & 19275.4 & 550199.3 & $22 \%$ \\
\hline & PM-10 (t) & 26372.7 & 19591.4 & 45964.1 & $12 \%$ \\
\hline & $\mathrm{SO} 2(\mathrm{t})$ & 416.4 & 695.2 & 1111.6 & $7 \%$ \\
\hline & Total AQCP (t) & 671532.0 & 71161.0 & 742693.0 & $20 \%$ \\
\hline \multirow{3}{*}{2014} & $\mathrm{CO} 2(\mathrm{kt})$ & 5554.2 & 2600.8 & 8155.0 & $11 \%$ \\
\hline & $\mathrm{CH} 4(\mathrm{t})$ & 1532.2 & 178.0 & 1710.2 & $11 \%$ \\
\hline & $\mathrm{N} 2 \mathrm{O}(\mathrm{t})$ & 153.2 & 106.8 & 260.0 & $11 \%$ \\
\hline
\end{tabular}


Ahmed, A. F. M.; Al-Kuwari, L;

Khazndar, A; Al-Marri, A

Assessment of Road Transport Atmospheric Emission of GHGs \& Criteria Pollutants

in Qatar: BAU Versus Paris

Agreement NDC Policy Scenarios

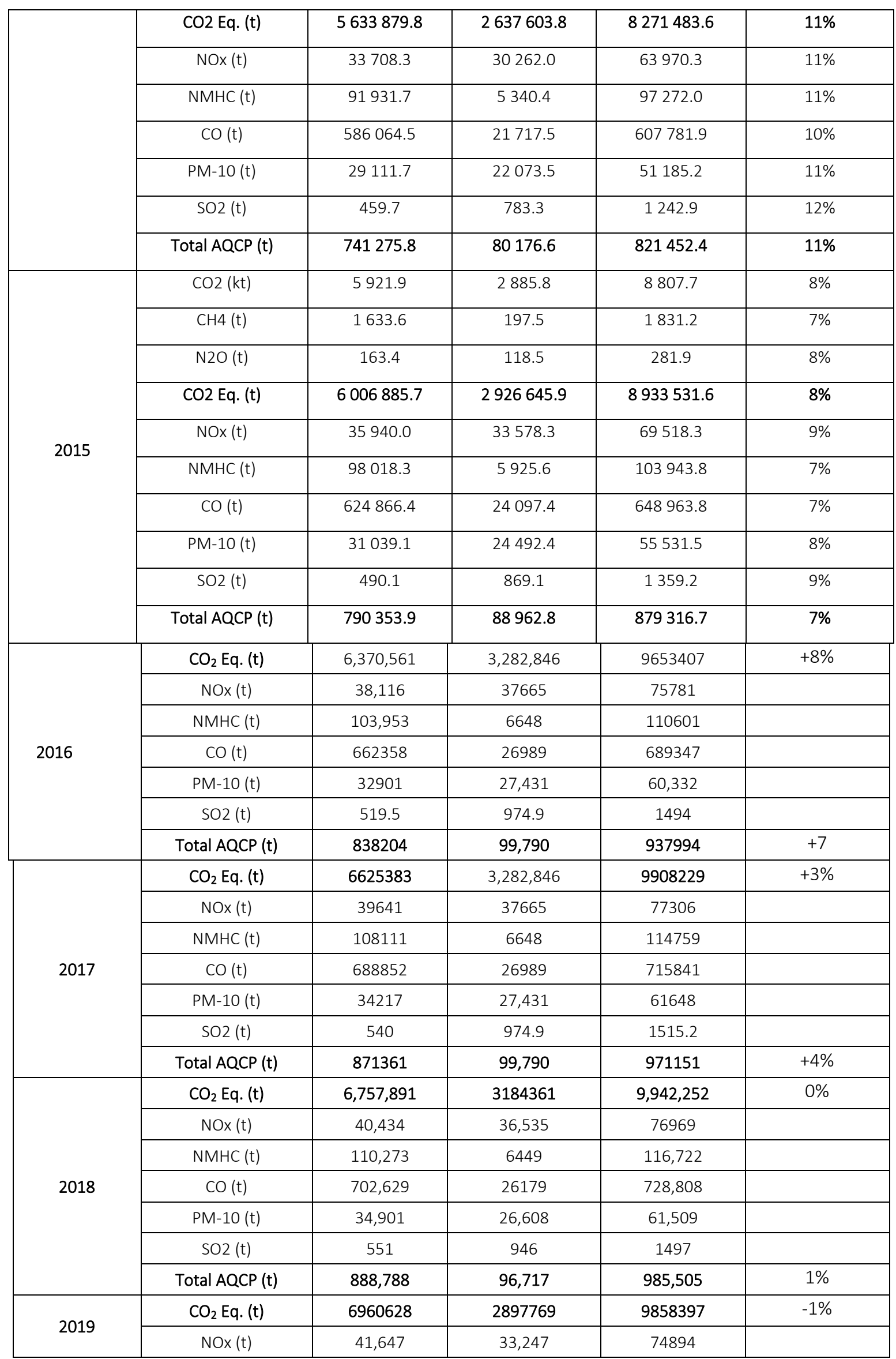


Ahmed, A. F. M.; Al-Kuwari, L;

Khazndar, A; Al-Marri, A
Assessment of Road Transport Atmospheric Emission of GHGs \& Criteria Pollutants

in Qatar: BAU Versus Paris

Agreement NDC Policy Scenarios

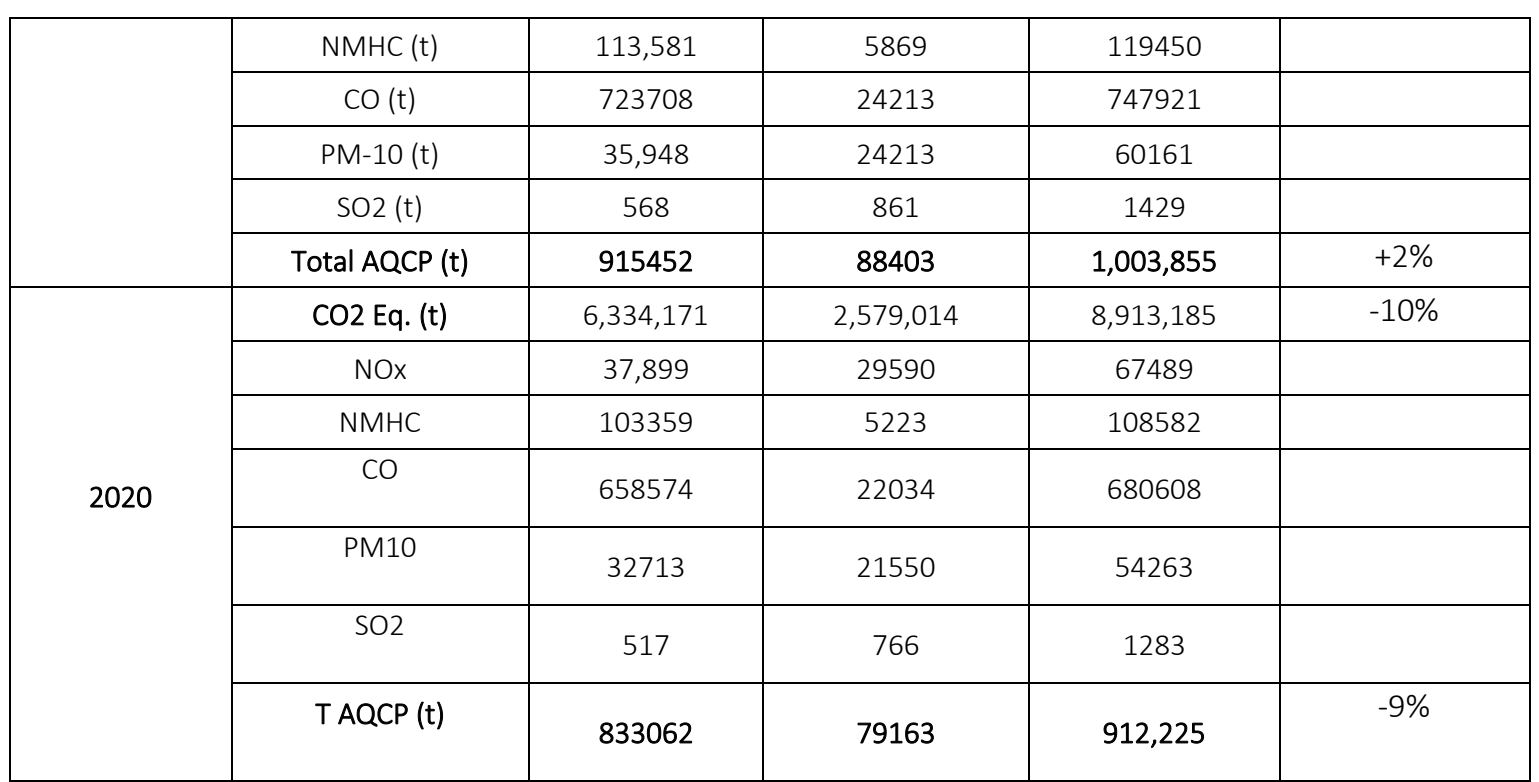

\begin{tabular}{|c|c|c|}
\hline \multirow{2}{*}{ Time Scale } & \multicolumn{2}{|c|}{ Cumulative Annual Emission Growth } \\
\cline { 2 - 3 } & GHGs Emission $\mathrm{CO}_{2}$-Eq. & AQCPs Emission \\
\hline $1995-2007$ & $+105 \%$ & +86 \\
\hline $2008-2015$ & $+74 \%$ & +84 \\
\hline $2016-2020$ & $-3 \%$ & $+2 \%$ \\
\hline
\end{tabular}

Table 3. Evolution of GHG\& Criteria Pollutant Emissions from Road Transport

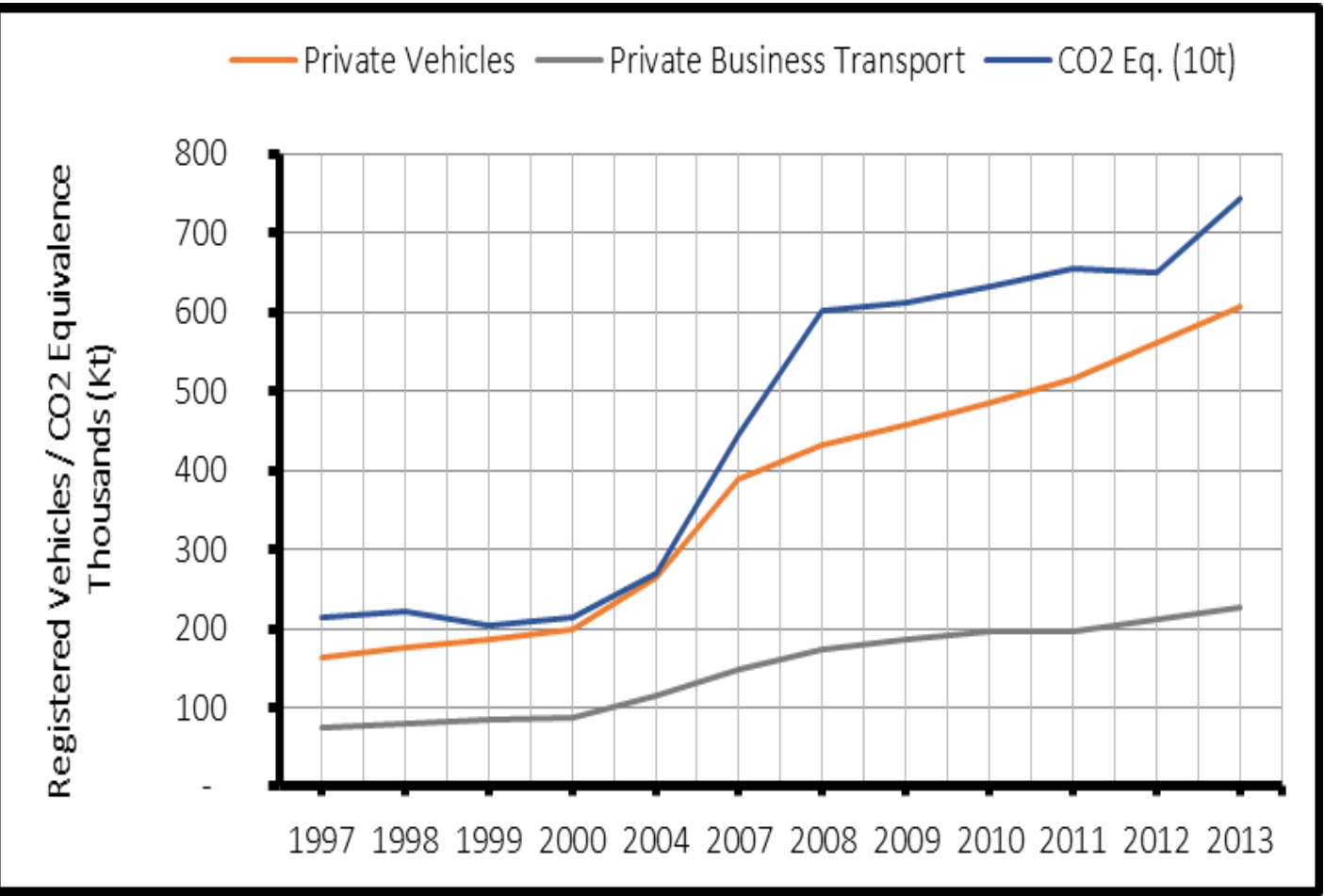

Figure 1. Evolution of registered vehicles \& GHG emissions 


\subsection{Socio-Economic Indicators}

Analysis of the historical trends of air emissions from road transport in Qatar against GDP (t/GDP) suggests that GHG emissions/GDP was maximum during 1996 - 2003 period, ranging between 100 to 120 t_CO Eq./million USD. From 2003 onward it started decresing untill it reached the lowest point in 2012 at 35 t_CO2 Eq./million USD. From 2013 to 2016 it increased reaching approximately 60 t_CO $C_{2} / G D P$ \& thereafter remained stable upto 2021. Business as usual (BAU) GHG emission scenario suggests that emission per GDP will stablize at $60 \mathrm{t} / \mathrm{GDP}$ during the timeframe 2021-2030. The policy emission scenario for the Nationally Determined Contribution is dicussed in the following section. A similar trend was obsrved for the AQCPs emissions per GDP. The maximum observed emissions were during $1995-2000$, reaching 12 t/GDP. The minimum occured in 2012 reaching 3 t/GDP. From $2015-2020$ the ratio reched 6 t/GDP and projected to remain consant upto 2030 based on BAU scenario (Figure 2).

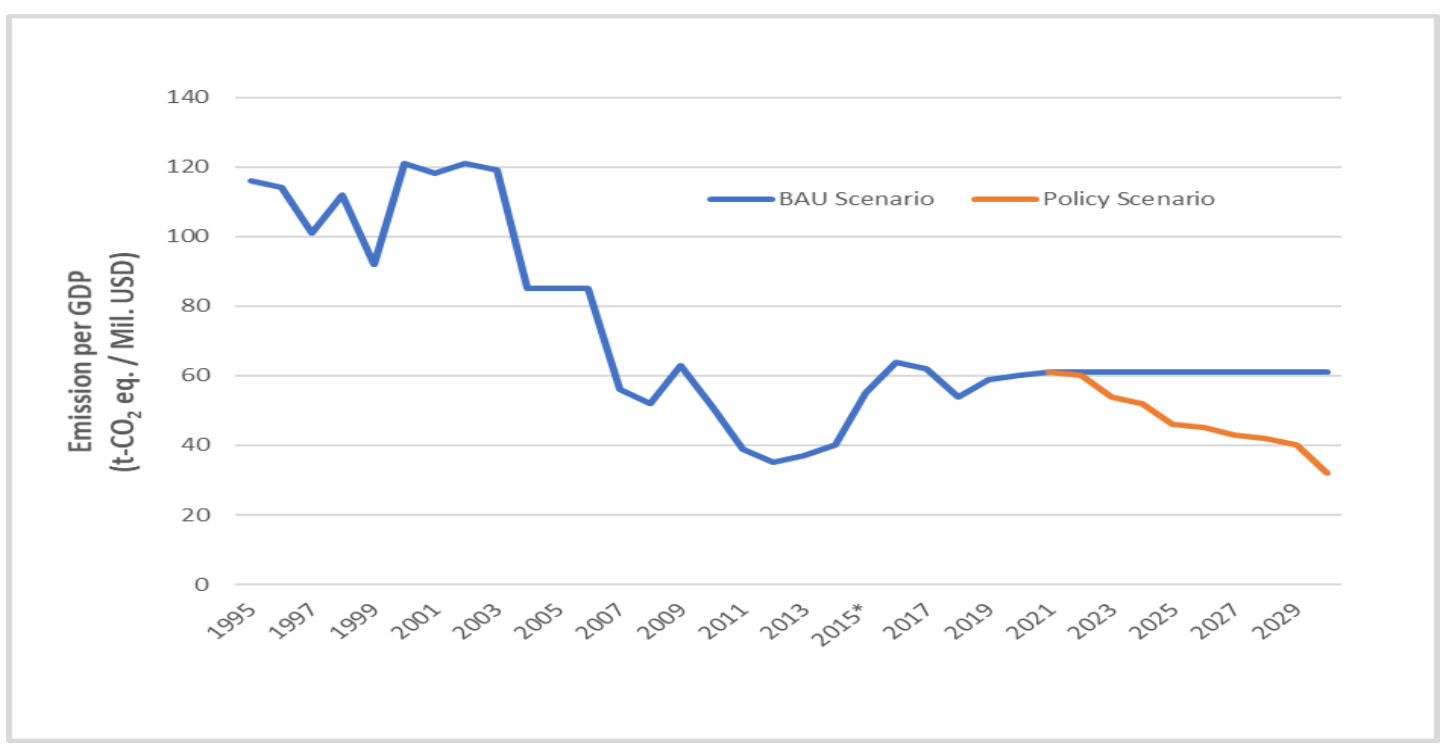

Figure 2. Historical trend of GHG emissions and GDP

\subsection{Qatar Paris Agreement NDC Commitment}

In this paper we examined the historical baseline BAU emission scenario for road transport within the context of Paris Agreement NDC. Qatar's emission reduction target for the timeframe 2020-2030 is evaluated in the lights of the planned national mitigation measures and policies designed to address the road transport emissions. Qatar nationally determined contribution (NDC) to the Paris Agreement has been submitted to UNFCCC in August 2021 (https://www.unfccc.int/sites). The communication reflects an enhanced ambition by the State to reduce its overall GHGs emission by $25 \%$ by the year 2030 relative to (BAU) baseline scenario. The transportation sector, including road transport, is included in this target. Measures to be implemented during the NDC timeframe included: replacement of the existing fleet of taxis (Mowasalat) with electric taxis (e-taxis) by 2030; replacement of the existing fleet of public transport and school buses of Mowasalat with electric buses by 2030; implementation of the Euro 6/VI standard 2023 onwards for light duty and heavy-duty vehicles \& regulations to allow new vehicle sales of only Euro 6/VI standard approved vehicles starting 2023; implementation of an incentive scheme from 2023 onwards to promote the penetration of electric vehicles (EVs); supporting investment required to set-up public charging infrastructure network for electric cars; implementing regulations to allow sale of only electric cars in Qatar after 2030; intensification of the public transportation network (buses, metro) to offer enhanced connectivity and to increase the share of public transportation in the modal split by 2030 
Ahmed, A. F. M.; Al-Kuwari, L;

Khazndar, A; Al-Marri, A
Assessment of Road Transport Atmospheric

Emission of GHGs \& Criteria Pollutants

in Qatar: BAU Versus Paris

Agreement NDC Policy Scenarios

resulting in reduced emission levels from private cars. Table 4 shows a summary of the GHG emission scenarios for both BAU \& NDC policy intervention described above. Figure 3 illustrates the historical GDP \& forecast up to 2030. The data was used to model emissions during the NDC timeframe 2020-2030, shown in Figure 4.

The results suggest that the road transport's NDC emission reduction ambition of the State of Qatar is significant (47\%) and as such exceeds the overall NDC objective of $25 \%$ by the year 2030. GHGs emissions from road transport is expected to reach under the BAU scenario about 13.5 million ton. Under the NDC target this will be reduced to about 7 million ton, a figure as low as the emission estimates in 2012. We expect a comparable reduction in the AQCPs emission as a result of the implementation of the NDC commitment. This aspect is currently being investigated.

Table 4. NDC Policy Intervention Emission Scenario 2021-2030

\begin{tabular}{|c|c|c|c|c|c|c|c|}
\hline \multirow{2}{*}{ Year } & \multirow{2}{*}{$\begin{array}{c}\text { GDP } \\
\text { Current } \\
\text { USD }\end{array}$} & \multirow{2}{*}{$\begin{array}{c}\text { BAU Emission } \\
\text { Scenario } \mathrm{CO}_{2} \\
\text { eq. }(\mathrm{K} \mathrm{t})\end{array}$} & \multirow{2}{*}{$\begin{array}{c}\text { Policy } \\
\text { Scenario } \\
\text { Emission } \\
\mathrm{CO}_{2} \text { eq. ( } \mathrm{K} \\
\mathrm{t} \text { ) }\end{array}$} & \multirow{2}{*}{$\begin{array}{c}\text { Intended } \\
\text { Mitigation } \\
\text { Quantity } \mathrm{CO}_{2} \\
\text { eq. (t) }\end{array}$} & \multirow{2}{*}{$\begin{array}{c}\text { Reduction } \\
\text { Relative to } \\
\text { BAU } \\
\text { Emissions }\end{array}$} & \multicolumn{2}{|c|}{$\begin{array}{l}\text { Emission per GDP t-CO2 eq./ } \\
\text { Million US \$ }\end{array}$} \\
\hline & & & & & & $\begin{array}{c}\text { BAU } \\
\text { Scenario }\end{array}$ & $\begin{array}{c}\text { Policy } \\
\text { Scenario }\end{array}$ \\
\hline 2021 & 165 & 10,046 & 10,046 & None & $0 \%$ & 61 & 61 \\
\hline 2022 & 171 & 10,411 & 10,342 & 69 & $1 \%$ & 61 & 60 \\
\hline 2023 & 177.1 & 10,782 & 9,546 & 1236 & $11 \%$ & 61 & 54 \\
\hline 2024 & 183.2 & 11,154 & 9,513 & 1641 & $15 \%$ & 61 & 52 \\
\hline 2025 & 189.4 & 11,531 & 8,743 & 2788 & $24 \%$ & 61 & 46 \\
\hline 2026 & 195.7 & 11,915 & 8,754 & 3161 & $27 \%$ & 61 & 45 \\
\hline 2027 & 202.0 & 12,298 & 8,752 & 3546 & $29 \%$ & 61 & 43 \\
\hline 2028 & 208.4 & 12,688 & 8,729 & 3959 & $31 \%$ & 61 & 42 \\
\hline 2029 & 214.9 & 13,084 & 8,641 & 4443 & $34 \%$ & 61 & 40 \\
\hline 2030 & 221.4 & 13,479 & 7,085 & 6394 & $47 \%$ & 61 & 32 \\
\hline
\end{tabular}


Ahmed, A. F. M.; Al-Kuwari, L;

Khazndar, A; Al-Marri, A
Assessment of Road Transport Atmospheric

Emission of GHGs \& Criteria Pollutants

in Qatar: BAU Versus Paris

Agreement NDC Policy Scenarios

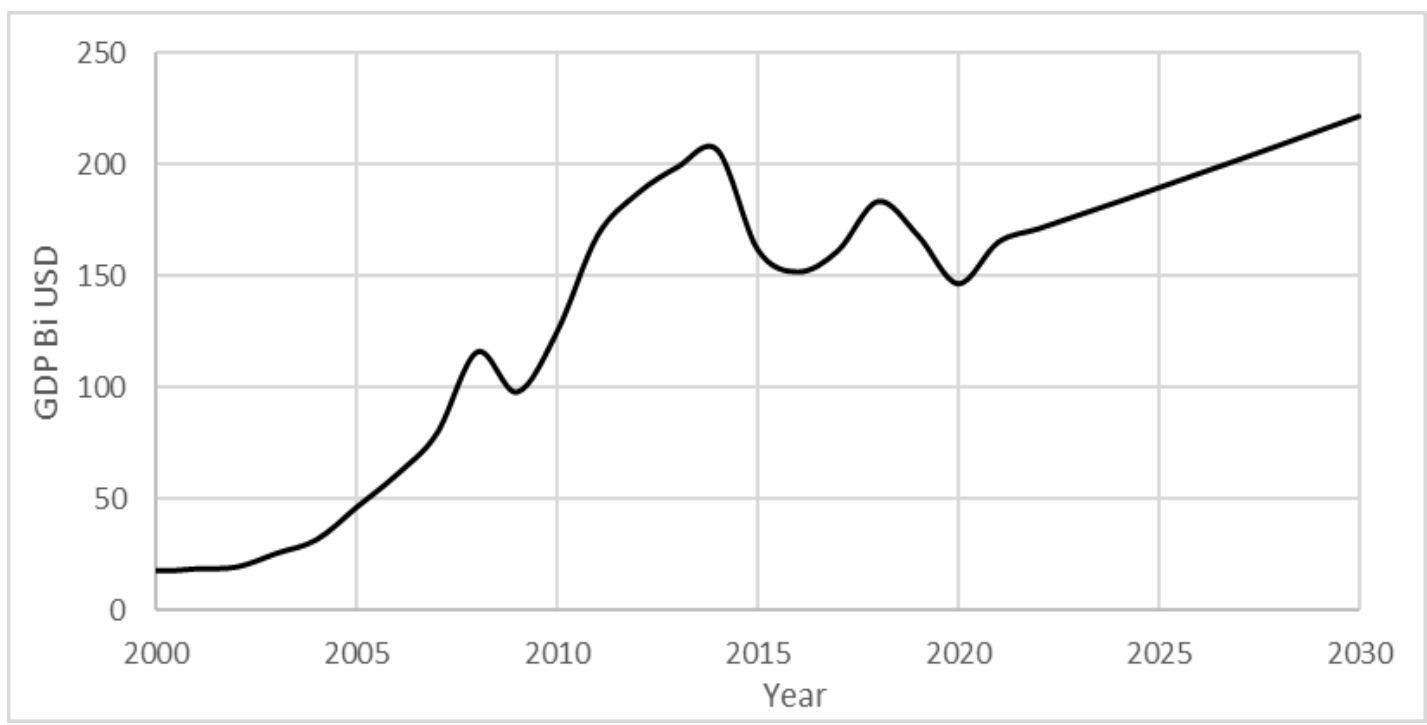

Figure 3. Historical \& Projected GDP of Qatar (Current Billion USD)

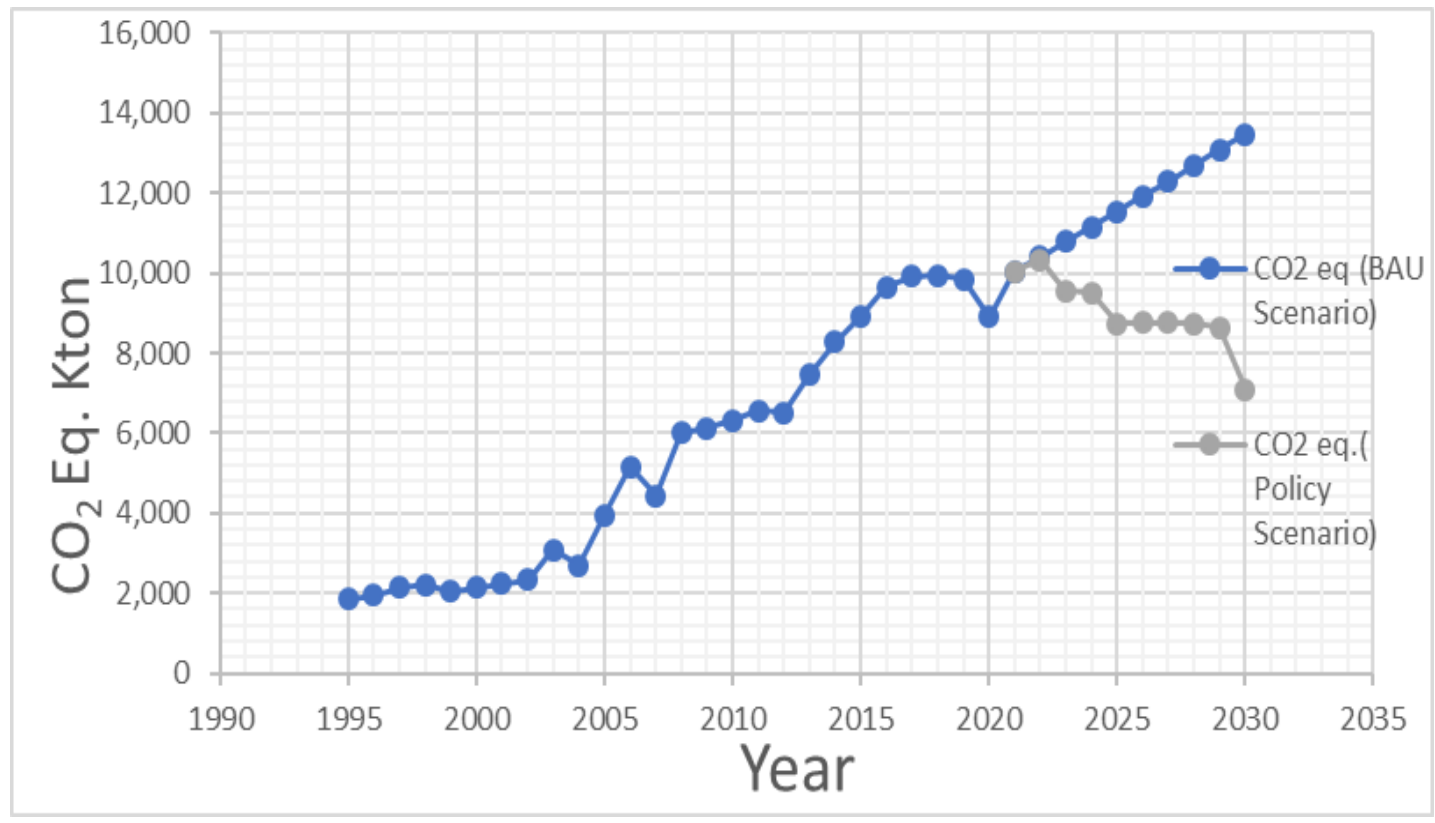

Figure 4. BAU (1995-2030) and Policy (2020-2030) Scenarios of GHGs Emissions 


\section{References}

1. EU GHG Guidelines (2007), COMMISSION DECISION of 18 July 2007 establishing guidelines for the monitoring and reporting of greenhouse gas emissions pursuant to Directive 2003/87/EC of the European Parliament and of the Council, https://eur-lex.europa.eu/legal-content/EN/

2. EU Air Quality Policy \& WHO Guideline Value for Health (2014): http://www.europarl.europa.eu/studies;

3. IPCC Guideline for National GHG Emissions (2006), Volume 2 Energy, TSU, IGES, Japan, https://www.ipcc-nggip.iges.or.jp/support/Primer 2006GLs.pdf

4. IPCC Good Practice Guidance and Uncertainty Management in National Greenhouse Inventories (2000), Volume 2 Energy, TSU, IGES, Japan; https://www.ipcc.ch/publication/good-practice-guidanceand-uncertainty-management-in-national-greenhouse-gas-inventories/

5. Revised Guideline for National Greenhouse Inventories (1996), Volume 2 Energy, TSU, IGES, Japan; https://www.ipcc-nggip.iges.or.jp/meeting/pdfiles/2006GLs scoping meeting report final.pdf

6. US EPA Compilation for Air Pollutant Environmental Factors: Highway Mobile Sources (1985), AP 42, $4^{\text {th }}$ Edition, Annual Arbor, Michigan, USA; https://www.arlis.org/docs/vol2/hydropower/APA DOC no. 1762.pdf

7. Guy Hitchcock, Beth Conlan, Duncan Kay, Charlotte Brannigan \& Dan Newman June (2014) Air Quality \& Road Transport, RAC Foundation, London, UK;

https://www.racfoundation.org/assets/rac foundation/content/downloadables/racf ricardo aea air quality report hitchcock et al june 2014.pdf

8. Planning \& Statistics Authority,PSA (2021): https://www.psa.gov.qa/en;

9. National Fuel Company, Woqod (2019): https://www.qnb.com/sites/qnb/qnbfs/document/10-2019);

10. INC, Mo E (2011) https://www.unfccc.int/sites 